\title{
PREVALENCE OF OBSTRUCTIVE SLEEP APNEA IN AN INDIAN POPULATION: USING STOP- BANG QUESTIONNAIRE
}

\section{SNIGDHA PATTANAIK ${ }^{1 *}$, RAJAGOPAL R $^{2}$, NEETA MOHANTY ${ }^{3}$, SWATI PATTANAIK ${ }^{4}$}

${ }^{1}$ Department of Orthodontics and Dentofacial Orthopaedics, Institute of Dental Sciences, Siksha " 0 " Anusandhan (Deemed to be University), Bhubaneswar, Odisha, India. ${ }^{2}$ Department of Orthodontics and Dentofacial Orthopaedics, Saveetha Dental College, Saveetha Institute of Medical and Technical Sciences, Chennai, Tamil Nadu, India. ${ }^{3}$ Department of Oral and Maxillofacial Pathology and Oral Microbiology, Institute of Dental Sciences, Siksha "O" Anusandhan (Deemed to be University), Bhubaneswar, Odisha, India. ${ }^{4}$ Department of Community Dentistry, Institute of Dental Sciences, Siksha “0” Anusandhan (Deemed to be University), Bhubaneswar, Odisha, India. Email: dr.snigdhapattanaik@gmail.com

Received: 30 May 2018, Revised and Accepted: 27 June 2018

\section{ABSTRACT}

Objective: Obstructive sleep apnea (OSA) is a condition characterized by complete/partial obstruction of the upper airway that disrupts normal sleep pattern. It has become highly prevalent and negatively affects the quality of life. Reports show $\geq 4 \%$ of men and $\geq 2 \%$ of women, and mostly, the obese individuals are affected by OSA. OSA is independently associated with an increased likelihood of hypertension, cardiovascular disease, and diminished quality of life. Hence, it becomes a prime concern for health-care personnel to diagnose it at earliest. A screening tool is necessary to stratify patients based on their clinical symptoms, their physical examinations, and their risk factors. Thus, this study was taken up to assess the prevalence of OSA using the STOP-Bang questionnaire.

Methods: A total number of 1012 participants were selected using random sampling technique from various community health camps for the study. The participants were asked to fill in the STOP-Bang questionnaire. All questionnaire respondents were precisely briefed about this study in a face-toface interview. Data obtained from the survey were subjected to statistics, and descriptive analysis was done.

Results: The prevalence of OSA was found to be $13.7 \%$ by using the Stop Bang questionnaire. It was found that the prevalence of OSA was highest in the age group of 50-59 (21.7\%) and least in the age group of 18-29 (12.0\%). Gender-wise distribution of OSA based on the scoring was seen to be more, among males $(14.8 \%)$ and females showed a prevalence of $12.9 \%$.

Conclusion: This study concludes that the STOP-Bang method of screening showed a prevalence of $13.7 \%$. However, the prevalence of OSA did not show any significant difference in various age groups; it was found that males had a higher prevalence of OSA compared to females.

Keywords: Questionnaire survey, Obstructive sleep apnea, Prevalence, Snoring, Surveys and questionnaires.

(c) 2018 The Authors. Published by Innovare Academic Sciences Pvt Ltd. This is an open access article under the CC BY license (http://creativecommons. org/licenses/by/4. 0/) DOI: http://dx.doi.org/10.22159/ajpcr.2018.v11i11.27598

\section{INTRODUCTION}

Obstructive sleep apnea syndrome (OSAS) is a disorder of breathing during sleep characterized by prolonged partial upper airway obstruction or intermittent complete obstruction that disrupts normal sleep patterns and are often associated with arousals, sleep fragmentation, intermittent hypoxia, and hypercapnia including excessive daytime sleepiness, growth failure, school failure, behavioral problems or cor pulmonale, automobile accidents, or even sudden death. OSAS has long been recognized as a major contributor to morbidity and mortality in developed countries. The prevalence of OSAS based on the Wisconsin cohort study performed in the USA involving middle-aged adults $30-60$ years of age, is $2 \%$ in females and $4 \%$ in males (Young, 1993). According to a survey conducted on a semiurban Indian population, it was found that around $6.2 \%$ among total sample were diagnosed with high-risk OSAS and $33.5 \%$ of the obese population were at high risk of OSAS [1]

The public health impact of OSAS is now enormous in developing countries like India because of increasing urbanization as well as lifestyle modification and its potential contribution to the increased rates of cardiovascular disease and obesity. In 2006, a populationbased survey from North India had estimated the prevalence of OSAS at $3.6 \%$ (males and females being $4.9 \%$ and $2.1 \%$, respectively). They found the prevalence of OSA to be $13.7 \%$ [2]. There is not much data regarding the prevalence of OSA in the Indian population. Hence, this study was designed to study the prevalence of OSA by using the STOPBang questionnaire.

\section{METHODS}

The present study was approved by the Institutional Human Ethics Committee, Institute of Medical science and SUM Hospital, Bhubaneswar, Odisha (DMR/IMS-SH/SOA/10657). A written informed consent was obtained from all the participants after giving the information sheet and explaining the procedure. Permissions were obtained from the hospital authority of Institute of Medical Science and SUM Hospital for recruitment of participants for evaluation of OSA (July 2016-July 2017).

\section{Participants}

To collect the data, the questionnaires were distributed in various camps conducted by the Department of Public health Dentistry. They were explained about OSA and its effect on health and were asked to participate in the study. Interested participants were explained about the questionnaire survey in detail and were asked to fill the questionnaire. Finally, the data were collected from 507 male participants and 505 female participants who cooperative and were willing to be a part of the survey.

The final 1012 participants of the study population who were incused in the study were more than 18 years and did not have any history of major illness. The patients having any history of myocardial infarction, 
chronic obstructive pulmonary disease, and upper-airway surgery were excluded from the study. The participants were given STOP-Bang questionnaire by trained interviewers and were explained regarding it.

\section{Sample size and sampling technique}

The formula for the sample size with $(1-\alpha) \%$ confidence level and with maximum marginal error of estimate of " $\mathrm{d}$ " for constructing confidence interval of true prevalence using normal approximation has been used. The formula is as follows:

$\mathrm{N}($ Minimum Sample Size $)=\frac{z_{1-\propto / 2}^{2} P(1-p)}{d^{2}}$

Where $\mathrm{P}$ is a predetermined value of the prevalence of OSA that is ascertained by previous published data. $\alpha=$ the probability of rejecting the null hypothesis, when it is true is taken as $0.05,=$ standard normal value of $\mathrm{Z}$ is taken as 1.96 . $\mathrm{p}=30 \%, \mathrm{~d}=4 \%$ point in the absolute term, with this input, the minimum sample size n works out to be 505 .

Since the aim was to estimate the same among males and females. Our minimum sample size was 507 males and 505 females, and the total sample size was 1012

\section{Stop-bang questionnaire}

The STOP-Bang questionnaires were developed in response to the need for a concise, user-friendly OSA screening tool in pre-operative clinics. The STOP-Bang questionnaire includes the four questions used in the STOP questionnaire plus four additional demographic queries,20 for a total of eight dichotomous (yes/no) questions related to the clinical features of sleep apnea (snoring, tiredness, observed apnea, high blood pressure, body mass index (BMI), age, neck circumference, and male gender). For each question, answering "Yes" scores 1, a "No" response scores 0 , and the total score ranges from 0 to 8 . The components of STOP questionnaire were selected based on the factor analysis of 14 candidate questions designed to reflect snoring, daytime tiredness, observed breathing cessation, and high blood pressure. The "bang" items were chosen based on univariate analysis of item predictive performance [3].

The questionnaire can be completed quickly and easily (usually within 1-2 min), and overall response rates are typically high (90\%-100\%). The questionnaire has demonstrated a high sensitivity using a cutoff score of 3: 84\% in detecting any sleep apnea (apnea-hypopnea index $[\mathrm{AHI}]>5$ events $/ \mathrm{h}$ ), 93\% in detecting moderate-to-severe sleep apnea (AHI $>15$ events/h), and 100\% in detecting severe sleep apnea (AHI $>30$ events/h). Corresponding specificities were $56.4 \%, 43 \%$, and $37 \%$. If patients score $0-2$ on the STOP-Bang questionnaire, they are considered to be at low risk of OSA, and the possibility of those patients having moderate-to-severe sleep apnea can be confidently ruled out (Chung, 2008).

\section{Statistical analysis}

Sigma plot 13 (Systat software, USA) software was used to analyze the data collected through questionnaires. Descriptive statistics were done based on the demographic data. The association of age and gender with STOP-Bang questionnaire was done using Chi-square test. Results were presented with $95 \%$ confidence interval.

\section{RESULTS}

A total sample of 1012 participants, who reported to the hospital for a routine checkup, were included in the study, of which 507 were male and 505 were female. From these samples, 424 were in the age group of $18-29$ years, 166 were in the age group of 30-39 years, 183 were in the age group of 40-49 years, 124 were in the age group of 50-59 years, 83 were in the age group of 60-69 years and 32 were aged above 70 years.

In the present study, it was found that about $13.7 \%$ of the participants were diagnosed with OSA (Table 1). Out of the total participants who were diagnosed with OSA, 30 participants were having moderate, and 45 participants were having severe OSA (Graph 1). The STOP-Bang score was seen to be highest in the age group 50-59 years $(21.7 \%)$, followed by the age group of 40-49 years (15.8\%). STOP-Bang score for the age group of $18-29$ years was $12 \%$ and for $30-39$ years was $12.6 \%$. STOP-Bang score for the age group 70 and above is $12.5 \%$ (Table 2). This might be due to less number of participants in that age group. The Chi-square test did not show any statistically significant result in the association of OSA in various age group $(p=0.224)$.

Gender-wise distribution of OSA based on the STOP Bang was seen to be highest, among males (14.8\%) and females showed $12.9 \%$ (Graph 2). The Chi-square test did not show any statistically significant result in the association of OSA in different genders $(p=0.326)$.

\section{DISCUSSION}

OSA has turned out to be a relatively common condition occurring due to the obstruction of recurrent upper airway sleep. The development of negative intrapharyngeal during inspiration and collapse of the airway is prevented by the action of the pharyngeal abductor and dilator muscles [4]. Rhythmic activation of these muscles occurs during daytime respiration; however, during sleep, the muscles become hypotonic, and thus airway stability relies on pharyngeal size and pharyngeal tissue compliance, which leads to a reduction in dimension of airway

Table 1: Prevalence of sleep apnea

\begin{tabular}{lll}
\hline Method & Sleep apnea & n (\%) \\
\hline OSA: STOP-Bang score & Absent & $874(86.3)$ \\
& Present & $138(13.7)$ \\
& Total & $1012(100.0)$ \\
\hline
\end{tabular}

$\%$ : Percentage, $\mathrm{n}=1012$

Table 2: Prevalence of sleep apnea in the different age group

\begin{tabular}{ll}
\hline Age group & Sleep apnea (STOP-Bang score) $\mathbf{n}(\%)$ \\
\hline $18-29$ & $51(12.0)$ \\
$30-39$ & $21(12.6)$ \\
$40-49$ & $29(15.8)$ \\
$50-59$ & $18(21.7)$ \\
$60-69$ & $15(12.1)$ \\
70 or more & $4(12.5)$ \\
Total & $138(13.7)$ \\
Chi-square, $\mathrm{p}$ & $\chi^{2}=3.01, \mathrm{df}=1, P=0.224$ \\
\hline
\end{tabular}

$\%$ : Percentage, $\mathrm{n}=1012$

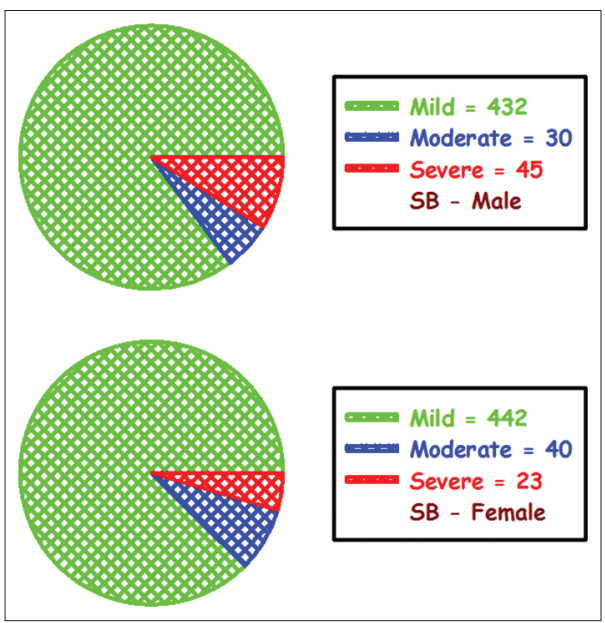

Graph 1: Prevalence of obstructive sleep apnea in different categories according to the STOP-Bang questionnaire. SB: STOPBang score, sample size: Male: 507, Female: 505 


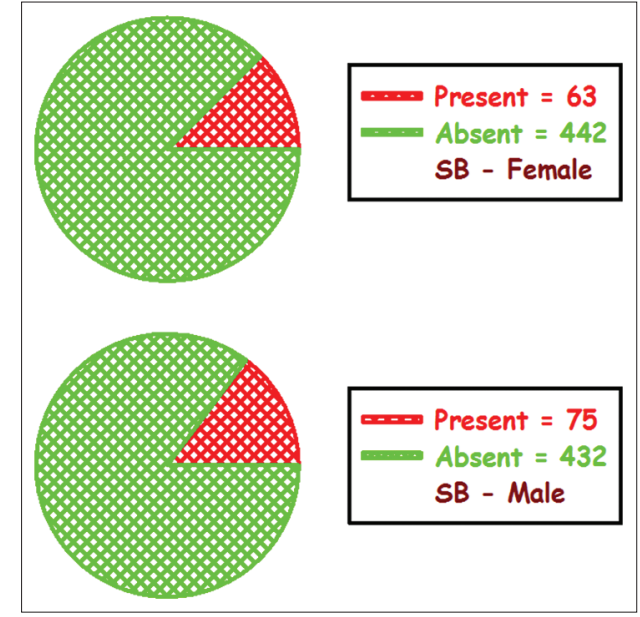

Graph 2: Prevalence of sleep apnea in different gender. SB: STOPBang Score. Sample size: Male: 507, Female: 505

paving the way for OSA. OSA is more prevalent in men (2-3 times) in comparison to women, and this has been linked to the pattern of fat distribution and difference in sex hormones.

Various chronic effects and hazardous comorbidities have been associated with OSA, if left undiagnosed. OSA is characterized by markedly elevated sympathetic nerve traffic while awake which may aid in chronic elevation of blood pressure. Increased left ventricular wall thickening has been observed in patients with OSA [5]. The left ventricular hypertrophy is an adverse prognosis of OSA and may also lead to impaired myocardial contractility [6]. Studies have suggested racial differences contribute to the pathogenesis of OSA, for example, when compared to Caucasians; Asians have shorter maxillae and mandible, smaller anterior-posterior facial dimensions, and lower BMI. This suggests that constriction of the rib cage is of greater importance than obesity and soft-tissue factors in the pathogenesis of OSA in Asians [7].

OSA, when left untreated, may lead to life-threatening consequences. Polysomnography remains "gold standard" for OSA diagnosis; however, unfortunately, it is expensive and has less accessibility. Hence, many questionnaire methods have been developed to screen OSA, such as American Society of Anesthesiologists, STOP questionnaire (snoring, tiredness, observed apnea, and high blood pressure), and STOPBang questionnaire (STOP including BMI, age, neck circumference, and gender), were validated on surgical patients; the Wisconsin questionnaire which was validated on patients selected from the general population, the Berlin questionnaire was validated in different target populations, i.e. sleep clinic patients, general population, and surgical patients, and Epworth sleepiness scale (ESS) which is a standard questionnaire to measure daytime sleepiness [8].

Various studies have been conducted for assessing the prevalence in different ethnic and racial groups; however, most of these studies were limited to the white population. Hence, the global prevalence of OSA cannot be reliably compared. In a study on a group of the Chinese population, it was found that there was the prevalence OSA in the Chinese population is only $4 \%$ similar to the Western studies [9]. Another study showed that East Asian men were less obese but had a [10] greater severity of OSA. It was also found that the prevalence of OSA is more in the Urban Indian population. It was $7.5 \%$ higher than the rates obtained from other epidemiological studies done across the globe [11].

A population-based study from North India estimated that the prevalence of OSAS at 3.6\% and OSA to be $13.7 \%$ [2]. A study from South Delhi reported the prevalence of OSA to be $9.3 \%$ and OSAS to be $2.8 \%$ [12]. Another hospital-based study from North India estimated the prevalence of OSA and OSAS to be $4.4 \%$ and $2.4 \%$ in males and $2.5 \%$ and $1 \%$ in females [13]. The prevalence of OSA in the Indian population is three-fold higher in men as compared to women (Reddy, 2009). According to a study by Singh et al., in North Indian population, it was found that $6.2 \%$ of the total populations were at high risk of OSAS whereas $33.5 \%$ of the obese population was at high-risk OSAS [1]. In our study, the prevalence of OSA was found to be $13.7 \%$ according to the STOP-Bang score which may be attributed to the higher BMI and presence of central obesity in this population (Table 1).

In an earlier study, it was found that the STOP-Bang questionnaire has high sensitivity for detecting OSA for moderate and severe OSA (93\% and $100 \%$, respectively), yet the specificity at the same cutoff of the STOP-Bang questionnaire was still low: $47 \%$ and $37 \%$ for moderate and severe OSA, respectively, resulting in fairly high false-positive rates [3]. Silva et al. reported that the STOP-Bang questionnaire had the highest sensitivity for moderate-to-severe $(87.0 \%)$ and severe sleep-disordered breathing (SDB) $(70.4 \%)$ in comparison to the ESS and the STOP questionnaire. STOP and STOP-Bang questionnaires had the advantage of being easily scored. Moreover, they were considered the most accurate questionnaires for OSA screening in surgical patients [14]. In another study done by El-Sayad, it was seen that the sensitivity of the STOP-Bang questionnaire was again higher compared to Berlin and STOP Questionnaire. The sensitivity recorded for moderate-to-severe SDB and severe SDB was $95.07 \%$ and $91.97 \%$, respectively [15].

The present study is the first population-based survey of the prevalence of OSA done in Odisha. In this study, the prevalence of OSA could be clearly related to the increase in age. OSA was more prevalent in the older age group of $50-59$ years $(21.7 \%)$. It was similar to a study done by Marques et al. which shows the highest prevalence with the mean age of 56 years. This clearly indicates that age being a strong risk factor for SDB over the middle decades of life (Table 2).

The present study also compared gender difference with respect to the prevalence of OSA. The prevalence of OSA was seen to be highest, among males $(14.2 \%)$ and females showed a prevalence rate of $11.7 \%$ (Graph 2). The male-to-female ratio is estimated between 3:1 and 5:1 in the general population and at 8:1-10:1 in some clinical populations [16].

Early clinical observations by Wilhoit and Suratt noted that OSA was quite rare in premenopausal women in comparison to postmenopausal women, attributing the disease to craniofacial abnormalities and severe obesity [17]. Bixler et al. studied about pre and postmenopausal women and OSA prevalence. They concluded that OSA prevalence is comparatively higher in postmenopausal than premenopausal women [18]. Compared with premenopausal women, the postmenopausal group was 3.5 times more likely to have SDB defined by an AHI of 15 or more, indicating that menopause was an independent risk factor for the development of SDB [19].

Obesity is considered to be one of the major risk factors for OSAS. In obese clinical patients, the prevalence of OSAS has been reported to be as high as $50 \%-80 \%$ whereas $60 \%-90 \%$ of OSAS patients may be overweight [20]. In large epidemiological studies, the association between overweight and OSAS in the general population has been evaluated, such as Wisconsin Sleep Cohort Study where obesity was found to be a significant risk factor for an AHI of $\geq 5$, and a single unit increase in BMI was associated with a four-fold increase in OSA prevalence [21].

\section{CONCLUSION}

From the present study, it can be concluded that the prevalence of OSA is considerably high with noticeable variation and increase in age and gender. Males are more prevalently affected by OSA in comparison to females. However, the statistically significant difference was not found in the association of OSA about age and gender. 


\section{AUTHORS' CONTRIBUTIONS}

Dr. Snigdha Pattanaik: Study design, data collection, and analysis, manuscript preparation. Dr. R Rajagopal: Study design and concept. Dr. Neeta Mohanty: Study design and concept. Dr. Swati Pattanaik: Statistical analysis.

\section{CONFLICTS OF INTERESTS}

Nil.

\section{REFERENCES}

1. Singh A, Prasad R, Garg R, Kant S, Hosmane GB, Dubey A, et al. A study to estimate prevalence and risk factors of obstructive sleep apnoea syndrome in a semi-urban Indian population. Monaldi Arch Chest Dis 2017;87:773.

2. Sharma SK, Kumpawat S, Banga A, Goel A. Prevalence and risk factors of obstructive sleep apnea syndrome in a population of Delhi, India. Chest 2006; 130:149-56.

3. Chung F, Abdullah HR, Liao P. STOP-bang questionnaire: A Practical approach to screen for obstructive sleep apnea. Chest 2016;149:631-8.

4. Akanbi MO, Agaba PA, Ozoh OB, Ocheke AN, Gimba ZM, Ukoli CO, et al. Obesity and obstructive sleep apnea risk among Nigerians. J Med Trop 2017;19:110-5.

5. Pinto JA, Ribeiro DK, Cavallini AF, Duarte C, Freitas GS. Comorbidities associated with obstructive sleep apnea: A Retrospective study. Int Arch Otorhinolaryngol 2016;20:145-50.

6. Zhang W, Si LY. Obstructive sleep apnea syndrome (OSAS) and hypertension: Pathogenic mechanisms and possible therapeutic approaches. Ups J Med Sci 2012;117:370-82.

7. Palmer LJ, Buxbaum SG, Larkin E, Patel SR, Elston RC, Tishler PV, et al. A whole-genome scan for obstructive sleep apnea and obesity. Am J Hum Genet 2003;72:340-50.

8. Peppard PE, Young T, Barnet JH, Palta M, Hagen EW, Hla KM, et al. Increased prevalence of sleep-disordered breathing in adults. Am J Epidemiol 2013;177:1006-14.

9. Ip MS, Lam B, Lauder IJ, Tsang KW, Chung KF, Mok YW, et al.
A community study of sleep-disordered breathing in middle-aged Chinese men in Hong Kong. Chest 2001;119:62-9.

10. Li KK, Powell NB, Kushida C, Riley RW, Adornato B, Guilleminault C, et al. A comparison of Asian and white patients with obstructive sleep apnea syndrome. Laryngoscope 1999;109:1937-40.

11. Udwadia ZF, Doshi AV, Lonkar SG, Singh CI. Prevalence of sleepdisordered breathing and sleep apnea in middle-aged urban Indian men. Am J Respir Crit Care Med 2004;169:168-73.

12. Reddy EV, Kadhiravan T, Mishra HK, Sreenivas V, Handa KK, Sinha S, et al. Prevalence and risk factors of obstructive sleep apnea among middle-aged urban Indians: A community-based study. Sleep Med 2009;10:913-8.

13. Vijayan VK, Patial K. Prevalence of obstructive sleep apnoea syndrome in Delhi, India. Chest 2006;130:149-56.

14. Nagappa M, Liao P, Wong J, Auckley D, Ramachandran SK, Memtsoudis S, et al. Validation of the STOP-bang questionnaire as a screening tool for obstructive sleep apnea among different populations: A Systematic review and meta-analysis. PLoS One 2015;10:e0143697.

15. El-Sayed IM. Comparison of four sleep questionnaires for screening obstructive sleep apnea. Egypt J Chest Dis Tuberc 2012;61:433-41.

16. Young T, Palta M, Dempsey J, Skatrud J, Weber S, Badr S, et al. The occurrence of sleep-disordered breathing among middle-aged adults. N Engl J Med 1993;328:1230-5.

17. Wilhoit SC, Suratt PM. Obstructive sleep apnea in premenopausal women. A comparison with men and with postmenopausal women. Chest 1987;91:654-8.

18. Bixler EO, Vgontzas AN, Lin HM, Ten Have T, Rein J, Vela-Bueno A, et al. Prevalence of sleep-disordered breathing in women: Effects of gender. Am J Respir Crit Care Med 2001;163:608-13.

19. Young T, Finn L, Austin D, Peterson A. Menopausal status and sleepdisordered breathing in the Wisconsin sleep cohort study. Am J Respir Crit Care Med 2003;167:1181-5.

20. Oo SS, Rao US, Zin T. Prevalence and factors associated with obesity among adult at the Kampung Kolam, east coast Malaysian peninsula-a cross sectional study. Int J Pharm Pharm Sci 2017;9:273-81.

21. Bollapragada MK, Shantaram M, Kumar RS. Obesity: Development, epidemiology, factors affecting, quantity, health hazards, management and natural treatment-a review. Int J Pharm Pharm Sci 2017;9:12-26. 